\title{
Review Seminar Based on CRS in Introductory Physics
}

\author{
Yu Jie Chen, Mi Su, Zhi Xin Huang* \\ College of Physical Science and Technology, Central China Normal University, Wuhan, China \\ Email address: \\ 1184557939@qq.com (Yu Jie Chen),369951909@qq.com (Mi Su), huangzx@mail.ccnu.edu.cn (Zhi Xin Huang) \\ ${ }^{*}$ Corresponding author
}

\section{To cite this article:}

Yu Jie Chen, Mi Su, Zhi Xin Huang. Review Seminar Based on CRS in Introductory Physics. Science Journal of Education. Vol. 8, No. 4, 2020, pp. 108-113. doi: 10.11648/j.sjedu.20200804.13

Received: August 3, 2020; Accepted: August 18, 2020; Published: September 8, 2020

\begin{abstract}
The research on the relationship between the teaching model of seminar and the learning gain of students has been a hot topic for a long time. As the Classroom Response System (CRS) is widely used in interactive teaching environments to attract students by asking questions. This paper takes electromagnetism of introductory physics as an example to study the teaching mode of review seminar based on CRS. Focusing on the necessity of review seminar based on CRS, the teaching efficiency as well as the stability of students' learning effect under this teaching mode, this paper qualitatively discusses the necessity and teaching efficiency of review seminar based on CRS, and quantifies the learning effect of students under this mode from the correct transformation of students' concept and its stability. The results show that the teaching model of seminar based on CRS is suitable for the teaching of problem sets in introductory and has high teaching efficiency. Most of the students have the right change of physics concept after the discussion with their peers, and have stable learning gain. At the end of the paper, we discuss the shortcomings of the research and looks forward to the future research direction.
\end{abstract}

Keywords: Classroom Response System, Review Seminar, Introductory Physics

\section{Introduction}

Seminar is a teaching mode that integrates research-based learning, autonomous learning, and peer learning [1]. Studies have shown that the application of the teaching model of seminars in introductory physics can improve students' interest in physics knowledge and interpersonal skills while improving teaching quality [2-4]. Classroom Response System (CRS) is a kind of technology application that can effectively mobilize students' active participation and interaction in Classroom teaching and has the function of immediate feedback [5-7]. Research shows that the combination of the second-generation CRS using Clicker with the general discussion classroom teaching mode and rational application in the introductory physics classroom can improve students' attendance [8] as well as students' learning outcome [9-10], promote students to think about physics problems actively [11], deepen students' understanding of the problems [12], and thus improve students' academic performance [13-15]. With the development and popularization of wireless networks and intelligent mobility, a new generation of CRS, which combines Clicker and cloud platforms, is gradually being used in teaching. Can the
CRS-based seminar model be used in introductory physics review class? How effective is the CRS-based exercise seminar? How effective are the students? These are the problems that this paper pays attention to and it will take electromagnetism in introductory physics as an example to carry out research.

\section{Method}

\subsection{Design of the Study}

This research focuses on the students in the introductory physics class of Central China Normal University. The exercise class of this course uses the teaching mode of CRS-based exercise discussion. The study adopts qualitative analysis to discuss its teaching efficiency. It uses quantitative methods to study the learning effect of students. Firstly, it analyzes the in-class teaching effect of the classroom based on CRS's immediate feedback, and compares it with the final test to analyze the stability of its learning gain.

\subsection{Conduct of Research}

The teaching mode of the introductory physics review seminar based on CRS is mainly divided into two parts: 
preparation before class and seminar during class. First of all, students, teachers, and teaching assistants prepare for the corresponding classes. The teacher uploaded the resources such as the knowledge summary at the end of the chapter one week in advance. Taking "steady magnetic field" of the second electromagnetics of introductory physics (I) as an example, the teacher uploads the chapter summary resource of "Stable Magnetic Field" to the QQ group one week ago. Students download and review knowledge by themselves, and $\log$ in to a special website for testing within a specified time. On the one hand, the teaching assistants classify students according to their initial mastery of this chapter according to the rules of homogeneity between groups and heterogeneity within the groups; on the other hand, organize and report the students' mastery to the teachers. Based on the feedback, the teacher determines the difficult and error-prone points that students still have, and then determines the problem sets that the student needs to discuss, as shown in Table 1, which is added to the CRS voting questionnaire activity by the teaching assistant, then the teaching assistant adds them to the voting questionnaire of CRS in advance.

Table 1. Discussion Questions of "Stable Magnetic Field".

\begin{tabular}{ll}
\hline Existing Difficulties and Prone Points for Students & \multicolumn{1}{l}{ Item } \\
\hline Current intensity, application of Biot-Savar's law, principle of magnetic induction superposition & 1 \\
Application of Biot-Savart's Law & 2 \\
Magnetic flux & 3 \\
Understanding of the Ampere Loop Theorem & 5 \\
Application of Ampere Loop Theorem & 6 \\
Knowing the direction of the magnetic field and the effect of the magnetic field on the current-carrying wire with known current \\
Effect of magnetic field on moving charge, magnetic moment, magnetic moment \\
Magnetic field strength vector, ampere loop theorem in magnetic medium \\
\hline
\end{tabular}

The discussion process in the class is shown in figure 1 below, which is mainly divided into three major links. First, the teacher guides students to review the important knowledge that needs to be discussed in the chapter according to the content prepared before the class. Secondly, the teacher guides the students to review, test and consolidate the knowledge learned in this chapter through problem discussions. The teaching assistant first shows the seminar questions arranged by the teacher before the class, and then operates the classroom response system based on Clicker and Moso Teach platform to conduct real-time detection of students' learning content. At the same time, students should think independently within the specified time, and then participate in the detection of CRS on the mobile phone terminal. The CRS shows that the feedback of the students' first real-time detection of "Stable Magnetic Field" is shown in Table 2. If the correct feedback rate of the first instant detection is between $30 \%$ and $70 \%$, such as the steady magnetic field discussion question 1 , the students need to conduct peer discussion according to the group before class within the specified time, and then perform the second real-time detection. The teaching assistant then guides the students to explain the questions based on the answers and the changes in the two answers, and then moves on to the discussion of the next question. If the CRS shows that the accuracy rate of the first instant vote is greater than $70 \%$, such as the steady magnetic field seminar question 2 , the student will be directly guided by the teaching assistant to analyze the question and move on to the next question. If the accuracy rate displayed by the CRS is less than $30 \%$, such as the stable magnetic field problem 8 , the teaching assistant should guide the students to analyze the concept of the problem before the discussion, and then conduct the CRS-based seminar. While the students are discussing, the teacher observes the students' discussions, and determines whether the knowledge points examined in this exercise need to be summarized according to the two response changes shown by the CRS. Finally, the teacher summarizes the existing mistake-prone points and difficult points of the students in the review seminar.

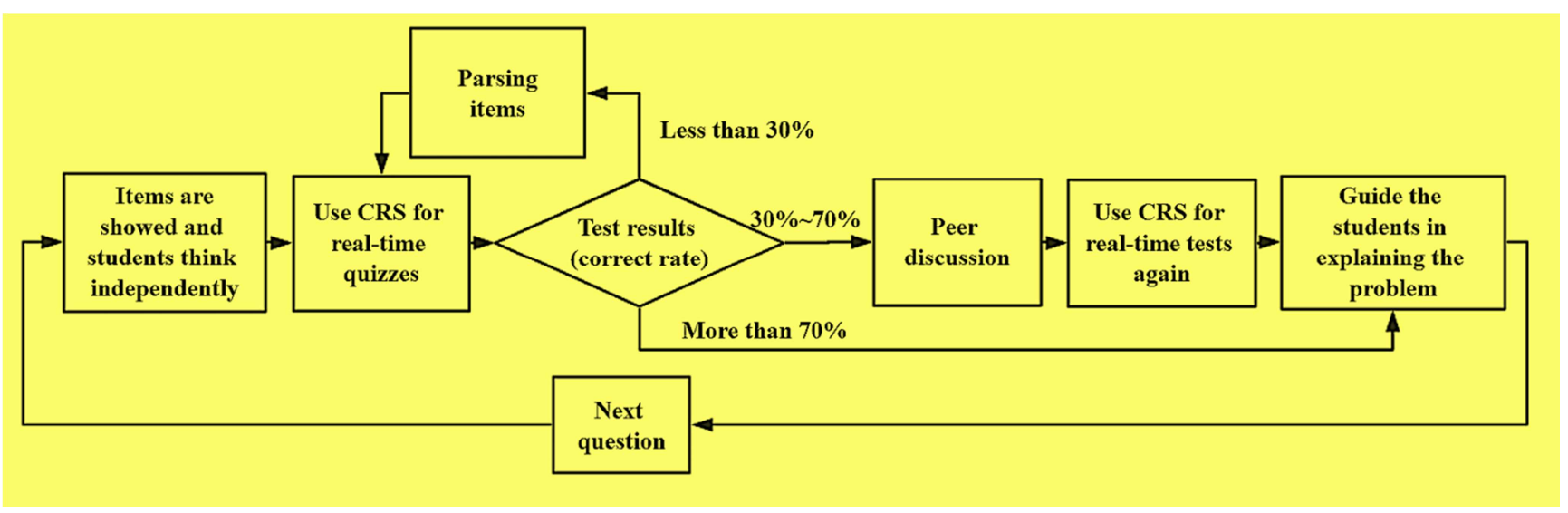

Figure 1. Discussion process in the CRS-based exercise seminar. 
Table 2. Results of the first real-time test of "Stable Magnetic Field".

\begin{tabular}{ll}
\hline Item & The first real-time test results \\
\hline 1 & $46 \%$ \\
2 & $83 \%$ \\
3 & $81 \%$ \\
4 & $85 \%$ \\
5 & $57 \%$ \\
6 & $87 \%$ \\
7 & $83 \%$ \\
8 & $23 \%$ \\
\hline
\end{tabular}

\subsection{Final Examination of the Same Knowledge Point}

In order to study the stability of student's learning gain, the research discusses and analyzes the results of the second real-time detection of the same knowledge point and the results of the final detection. To this end, the research classifies the knowledge points examined in the exercises to be studied, and selects four knowledge points: Abe's loop theorem, magnetic field strength, Gauss's theorem, and magnetic field strength. In the final exam of introductory physics, it is examined in the form of single choice question, and the corresponding correct rate is calculated. The final exam question is a variant of the study problem, which is quite difficult.

\section{Results and Discussion}

\subsection{The Necessity of Applying CRS-based Review Teaching Mode}

First, use the CRS to count the correctness of the first real-time test results of the research questions in each chapter, and then analyze the average correct rate of the first real-time test in each chapter. The CRS immediate feedback of the average correct rate of the first instant detection of each chapter of the introductory physical electromagnetics is shown in Figure 2. The correct rate of each chapter is between $68.10 \%$ and $83.90 \%$. This shows that after self-preparation, classroom learning, and teacher-led review, students have a certain grasp of physics concepts, that is, students have a certain knowledge base; however, the average accuracy rate is not high, which indicates that despite the self-review and review of the chapter knowledge summary before the lesson and the self-testing on the website, the teacher guided the key and difficult points before the class discussions. there are still problems with the learning situation of students who have not grasped the concepts in place and have difficulty learning. Therefore, only by self-directed learning and teacher teaching, students can not break through the difficulties in knowledge acquisition. It can be seen that autonomous learning or teacher teaching at this time has little effect on the correct change of the concept of such students. Peer discussion can promote student-student communication and interaction. Through peer discussion, students' classroom participation and thinking of problems from other perspectives are promoted. Therefore, it is very necessary to carry out cooperative learning by using exercises

\subsection{Teaching Efficiency of Review Seminars Based on CRS}

After the students vote in the CRS on the mobile phone within a limited time, the teacher can get the information about the correct rate of the students' responses to the CRS instant feedback. Based on the immediate feedback effect of CRS, the teacher can directly judge the students' mastery of the knowledge points examined in the research question by the percentage of the test results, and then choose the appropriate learning mode. When the accuracy of the first test is greater than $70 \%$, most of the students have mastered the knowledge and adopt an autonomous learning mode. However, when the accuracy is between $30 \%$ and $70 \%$, some students have difficulties in learning the knowledge points, so they adopt the cooperative learning mode of peer discussion. However, if the accuracy rate is less than $30 \%$, most of the students have difficulties in learning the knowledge point, so teachers' guidance and advice should be added on the basis of peer discussion. The course of the traditional introductory physics exercise class is that the teacher first displays the topic, then reserves time for students to think and solve the problem, and finally guides and comments. On the one hand, without the application of CRS, teachers cannot obtain the mastery of students; on the other hand, only the interaction between teachers and students, without the interaction between students and students, the interaction and participation of students are not high, and the teaching efficiency is compared low. Compared with traditional introductory physics problem classes, the CRS-based review seminars are more targeted and directional. There are corresponding scientific learning models for problems of different difficulties, which avoid the ineffective teaching of full teaching and improves the teaching efficiency.

\subsection{Learning Effects of Review Seminars Based on CRS}

For each chapter, the accuracy rate of the first real-time detection is less than $70 \%$, that is, the accuracy rate of the two real-time detection and the increase rate of the exercise requiring peer discussion are statistically analyzed. The CRS shows the results as shown in Table 3 below. After discussion, the correct rate of each question has been greatly improved, with the increase rate of correct rate ranging from $44.9 \%$ to $252.2 \%$. This indicates that the physics concepts of most students with learning difficulties have undergone correct changes after discussion. Among them, the part of the discussion topic and examines the knowledge points as shown in Table 4, such as the vacuum electrostatic field in the discussion item 1, examines hooke's law and the integrated use of coulomb's law, its correct overall increase from $69 \%$ to $100 \%$, after discussion, all the hooke's law and coulomb's law learning difficulties of students in the classroom have taken place in the concept of right shift. For item 14, the increase rate of correct rate after peer discussion reached $139.4 \%$, 
indicating that more than half of the students broke the learning barrier of dielectric polarization. It is worth noting that in item 24, the correct answer rate of the first real-time detection result is $23 \%$, that is, students have great difficulties in learning the magnetic field intensity vector and ampere loop theorem in magnetic medium, and the teaching assistant needs to analyze and select the concept of this question. The immediate detection results increased from $23 \%$ to $81 \%$, an increase of $58 \%$, and an increase rate of $252 \%$. This indicates that, for the problem, the guidance of the teaching assistant to the peer discussion among students is more conducive to the correct transformation of students' concepts. The above analysis shows that after peer discussion, most of the students have undergone correct conceptual changes and gained learning benefits. CRS-based exercise discussion has the advantage of promoting student learning.

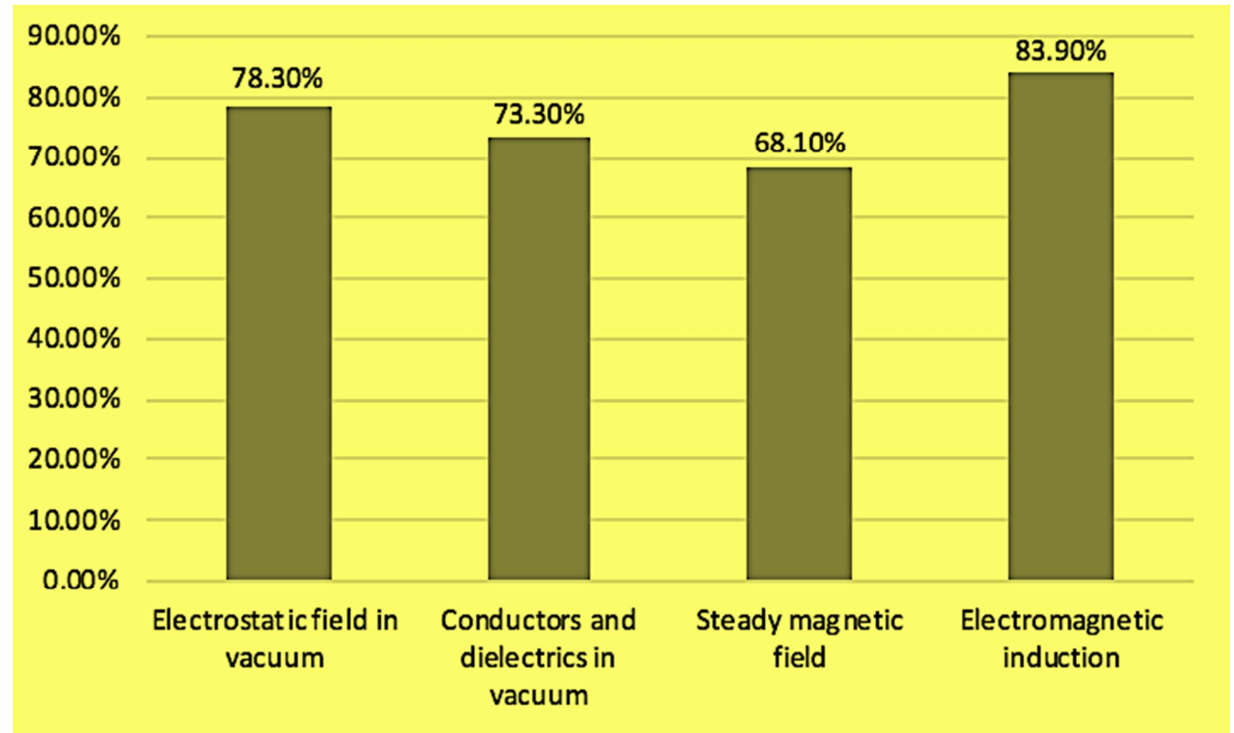

Figure 2. The first real-time test results of the problem sets in each chapter of electromagnetism.

Table 3. The correct rate and increase rate of the two real-time tests for electromagnetics.

\begin{tabular}{llll}
\hline Item & The first real-time test results & The second real-time test results & Increase rate \\
\hline 1 & $69 \%$ & $100 \%$ & $44.9 \%$ \\
4 & $51 \%$ & $93 \%$ & $82.4 \%$ \\
8 & $54 \%$ & $85 \%$ & $57.4 \%$ \\
10 & $56 \%$ & $85 \%$ & $51.8 \%$ \\
14 & $33 \%$ & $79 \%$ & $139.4 \%$ \\
17 & $46 \%$ & $85 \%$ & $84.5 \%$ \\
21 & $57 \%$ & $94 \%$ & $64.9 \%$ \\
24 & $23 \%$ & $81 \%$ & $252.2 \%$ \\
27 & $52 \%$ & $87 \%$ & $67.3 \%$ \\
\hline
\end{tabular}

Table 4. Part of the questions and its examination knowledge.

\begin{tabular}{|c|c|}
\hline Questions & Examination knowledge \\
\hline $\begin{array}{l}\text { Item 1: On the level of smooth insulation, there are two identical balls with the same amount of charge, connected by a } \\
\text { light insulating spring. At rest, the spring extends by X1; If you cut the charge of both balls in half, the spring will } \\
\text { extend by } X_{2} \text { when it comes to rest again. The following conclusions are correct () } \\
\text { A. } X_{2}=\frac{X_{1}}{2} \\
\text { B. } X_{2}=\frac{X_{1}}{4} \\
\text { C. } X_{2}>\frac{X_{1}}{4} \\
\text { D. } X_{2}<\frac{X_{1}}{4}\end{array}$ & Hooke’s law, coulomb's law \\
\hline $\begin{array}{l}\text { Item14: As for the polarization of the dielectric, the correct statement in the following is () } \\
\text { A. Dielectric molecules can be divided into non-polar molecules and polar molecules according to whether the positive } \\
\text { and negative charge centers of molecules coincide or not. Both of them are polarized in the outer electric field, and the } \\
\text { polarization process is the same } \\
\text { B. Displacement polarization refers to the phenomenon that the positive and negative charges appear on the surface of } \\
\text { the dielectric due to the slight relative displacement of the positive and negative center of the molecule under the action } \\
\text { of the external electric field, forming an electric dipole } \\
\text { C. The dielectric can also be excited to generate an electric field under the action of an external electric field, whose } \\
\text { strength can offset the external electric field, so the composite field strength inside the dielectric is zero } \\
\text { D. When the dielectric is uniformly polarized, there is no net polarization charge in the dielectric, and the polarization } \\
\text { charge is only distributed on the surface of the dielectric }\end{array}$ & Polarization of a dielectric \\
\hline
\end{tabular}




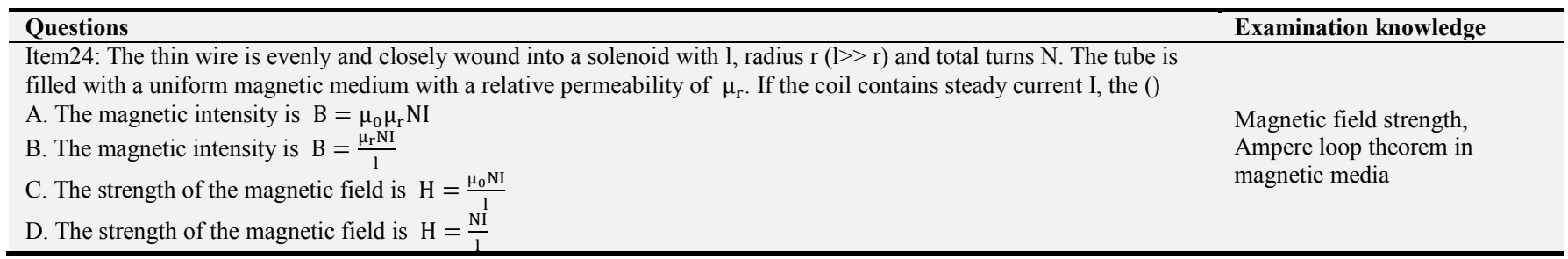

The immediate feedback from the CRS shows that the exercise discussion is beneficial to the correct transformation of students' physical concepts and helps to promote the students' grasp of knowledge. This is the immediate feedback of the learning effect of the students in the classroom. However, is the correct transformation of students' physical concepts a temporary product in the specific environment of the current seminar, or is it a stable learning benefit that is internalized into the correct concepts after discussion with peers? In order to explore the stability of the correct transformation of students' physical concepts, this article re-examines the knowledge points corresponding to the questions examined in the final exam, and discusses the differences between the two results through analysis of variance. If the $p$-value is greater than 0.05 , it means that there is no significant difference between the two grades, that is, the student's concept change is stable. In this paper, the four knowledge points of Abe's loop theorem, magnetic field strength, Gauss's theorem, and electromotive force are examined again in the form of multiple choice questions in the final exam of introductory physics. These four points corresponding exercises for discussion of the second instant detection and the accuracy of the final inspection as shown in Table 5, again for each knowledge point correspondence problem sets for the second time instant detection accuracy by the end of the university physics test with multiple choice test the accuracy of analysis of variance, as shown in Table 6, $P$ was 0.889 , greater than 0.05 , the same knowledge during real-time detection and final testing accuracy there was no significant difference, it shows that students' right of the concept of change is through a peer discussion after internalization of stable learning gains, based on the CRS of introductory physics exercises learning gains with stability of the seminar.

Table 5. Results of the second real-time test and the final test of the same knowledge point.

\begin{tabular}{lll}
\hline Examine knowledge points & Second real-time detection accuracy & Final detection accuracy \\
\hline Abe's Loop Theorem & $94 \%$ & $84 \%$ \\
Magnetic field strength & $81 \%$ & $88 \%$ \\
Gauss theorem & $93 \%$ & $89 \%$ \\
Electromotive force & $87 \%$ & $92 \%$ \\
\hline
\end{tabular}

Table 6. The second real-time test and the final test results of the variance analysis.

\begin{tabular}{llllll}
\hline SS & df & MS & F & P-value & F crit \\
\hline $5 \mathrm{E}-05$ & 1 & $5 \mathrm{E}-05$ & 0.02120 & 0.889 & 5.987378 \\
0.01415 & 6 & 0.00235 & & & \\
0.0142 & 7 & & & & \\
$5 \mathrm{E}-05$ & 1 & $5 \mathrm{E}-05$ & 0.02120 & 0.889 & 5.987378 \\
\hline
\end{tabular}

\section{Conclusion}

Through the above effect analysis, it can be seen that the review seminar model based on CRS can not only build a scientific and efficient review class, but also promote the transformation of most students' physics concepts and obtain stable learning benefits.

\subsection{It Makes the Review Class More Efficient}

This is due to the instant feedback effect with CRS and the superiority of peer discussion. On the one hand, by virtue of the advantages of immediate feedback of CRS, teachers can timely and correctly judge students' knowledge mastery, and guide students to adopt different learning modes according to the different ranges of the accuracy of the first real-time detection. Through the fusion of seminar and CRS, the teaching can be arranged more directionally and scientifically, so as to improve the efficiency of the class and reflect the dominant position of teachers.

\subsection{It Makes the Learning Benefits More Stable}

On the other hand, the first real-time test feedback of each chapter shows that some students still have wrong concepts after independent learning and testing before class, as well as teachers' key and difficult teaching. However, the learning mode of peer discussion promoted students to conduct cooperative learning, and the accuracy of the second real-time detection was greatly improved, that is, most students had the correct concept change after the discussion, resulting in learning benefits. It can be seen that for the students who still have difficulties in learning after reviewing, studying, and reviewing, peer discussion can promote students to overcome the difficulties more than the students' independent learning and the teacher's one-way guidance. Moreover, there was no significant difference in students' mastery of knowledge after the discussion of CRS feedback and the same knowledge points in the final examination. That is to say, the CRS-based review seminar does not change the physics concept of students temporarily, not the specific result of the current seminar, but the stable product of the students after the seminar. The correct transformation of physical concepts is stable, and the students' learning effect is significant and stable under the CRS-based 
review seminar model is remarkable and stable.

To sum up, the teaching mode of the review seminar based on CRS is a superior teaching mode. CRS-based review seminars can improve teachers' teaching efficiency and build a scientific and efficient college physics review class. At the same time, it can promote the correct transformation of students' concepts, and the students' learning effect is remarkable; there is no significant difference between the test results of the students after the discussion and the end-of-term test, and the learning income is stable. Therefore, the CRS-based review seminar is a teaching model suitable for introductory physics review classes.

CRS-based review seminars can effectively improve the learning benefits of students, which is beneficial to the whole students. But specific to a student, how the physical concepts has changed and to what extent, etc., are limited by the current CRS, and have not been solved yet. But it has important implications for our research. Therefore, how to optimize CRS, combine science and technology to track every student's conceptual change before and after peer discussion, as well as the internal and external factors of the change, so as to better improve classroom teaching efficiency and student learning effect, is the next step research direction.

\section{Fund Project}

This study is funded by the horizontal social science project of Central China Normal University (20202198092).

\section{References}

[1] Eric Mazur (2009). Farewell, Lecture?. Science. 2: 50-51.

[2] Zhu Hong, Ma Liping and Xiong Yu (2016). Study on the Effect of "Lecture in Large Class and Seminar in Small Class". China Higher Education Research, 01: 42-47.

[3] M. K. Smith. Why peer discussion improves student performance on in-class concept questions. Science. 2009 (2): 122-124.
[4] Risto Leinonen, Mervi A. Asikainen, and Pekka E. Hirvonen (2017). Peer discussions in lecture-based tutorials in introductory physics. Phys. Rev. Phys. Educ. Res. 13, 010114.

[5] Tolga Gok (2012). The impact of peer instruction on college students' beliefs about physics and conceptual understanding of electricity and magnetism. International Journal of Science and Mathematics Education, 10, 417-436.

[6] Chen Zhenzhen (2013). Designing and Instructing CRS-based College English Courses-A Case Study Using Socrative. Modern Educational Technology, 23 (10): 87-91.

[7] Kelly Miller, Nathaniel Lasry, Brian Lukoff, Julie Schell, and Eric Mazur (2014). Conceptual question response times in Peer Instruction classrooms. Phys. Rev. ST Phys. Educ. Res. 10, 020113.

[8] Caldwell, J (2007). Clickers in the large classroom: Current research and best-practice tips. Life Sciences Education, (1): 9.

[9] Kelly Miller, Julie Schell, Andrew Ho, Brian Lukoff, and Eric Mazur (2015). Response switching and self-efficacy in Peer Instruction classrooms. Phys. Rev. ST Phys. Educ. Res. 11, 010104 .

[10] Lasry, N (2008). Clickers or flashcards: ls there really a difference. Phys. Teacher, 46: 242-244.

[11] N. W. Reay, P. F. Li, L. Bao (2008). Testing a new voting machine question methodology. American Journal of Physics, Vol. 76 (2): 171-178.

[12] He Miaoman, Huang Zhixin and Zhou Dongying (2017). The preliminary study of seminar in college physics class based on cliker. Physics and Engineering, 27 (03): 45-51.

[13] Ryan Sayer, Emily Marshman, and Chandralekha SinghPhys (2016). Case study evaluating Just-In-Time Teaching and Peer Instruction using clickers in a quantum mechanics course. Rev. Phys. Educ. Res. 12, 020133.

[14] Nancy A Burnham, Snehalata V Kadam, Erin DeSilva (2017). In-class use of clickers and clicker tests improve learning and enable instant feedback and retests via automated grading. Phys. Educ. 52065018

[15] Neville W. Reay, Lei Bao, Peng fei Li (2005). Toward the effective use of voting machines in physics lectures. Am. J. Phys, 73 (6): 554-558. 